\title{
A Generalized Finite Hankel Type Transformation and a Parseval Type Equation
}

\author{
B.B.Waphare \\ Department of Mathematics, MITACSC, Alandi, \\ Savitribai Phule Pune University,Pune,India \\ balasahebwaphare@gmail.com
}

\begin{abstract}
In this paper, we study the finite Hankel type transformation on spaces of generalized functions by developing new procedure. Two Hankel type integral transformations $h_{\alpha, \beta}$ and $h_{\alpha, \beta}^{*}$ are considered and they satisfy Parseval type equation defined by (1.2). We have defined a space $S_{\alpha, \beta}$ of functions and a space $L_{\alpha, \beta}$ of complex sequences and it is further shown that $h_{\alpha, \beta}^{*}$ and $h_{\alpha, \beta}^{\prime}$ are isomorphisms from $S_{\alpha, \beta}$ onto $L_{\alpha, \beta}$ and $S_{\alpha, \beta}^{\prime}$ onto $L_{\alpha, \beta}^{\prime}$ respectively. Finally some applications of new generalized finite Hankel type transformation are established
\end{abstract}

Keywords: Finite Hankel type transformation, Parseval type equation, generalized finite Hankel type transformation.

2000 Mathematics subject classification: 46 F 12.

\section{INTRODUCTION}

I.N. Sneddon [14] was first to introduce finite Hankel transforms of classical functions. The same was later studied by [3], [4], [7], [15]. Recently Zemanian [18], Pandey and Pathak [11] and Pathak [12] extended these transforms to certain spaces of distributions as a special case of general theory on orthonromal series expansions of generalized functions. Dube [5], Pathak and Singh [13] and Mendez and Negrin [10] investigated finite Hankel transformations in other spaces of distributions through a procedure quite different from that one which was developed in [18] and [12].

We define finite Hankel type transformation of the first kind by

$$
\left(h_{\alpha, \beta} f\right)(n)=\int_{0}^{1} x J_{\alpha-\beta}\left(\lambda_{n} x\right) f(x) d x, \quad n=0,1,2, \ldots \ldots .
$$

for $(\alpha-\beta) \geq-\frac{1}{2}$, where $J_{v}$ denotes the Bessel function of the first kind and order $v$ and $\lambda_{n}$, $n=0,1,2, \ldots \ldots$, represent the positive roots of $J_{\alpha-\beta}(x)=0$ arranged in ascending order of magnitude $[17, \mathrm{p} .596]$.

For $(\alpha-\beta) \geq-\frac{1}{2}$ and $a \geq \frac{1}{2}$, we introduce the space $U_{\alpha, \beta, a}$ of finitely differentiable functions on $(0,1)$ such that

$$
\rho_{k}^{\alpha, \beta, a}(\phi)=\operatorname{Sup}_{0<x<1}\left|x^{a-1} B_{\alpha, \beta}^{* k} \phi(x)\right|<\infty \text {, for every } k \in \mathbb{N},
$$

where $B_{\alpha, \beta}^{*}=x^{-(\alpha-\beta)} D x^{4 \alpha} D x^{-(3 \alpha+\beta)}$.

$U_{\alpha, \beta, a}$ is equipped with the topology generated by the family of seminorms $\left\{\rho_{k}^{\alpha, \beta, a}\right\}_{k=0}^{\infty}$. Thus $U_{\alpha, \beta, a}$ is a Frechet space. $U_{\alpha, \beta, a}^{\prime}$ denotes the dual of $U_{\alpha, \beta, a}$ and is endowed with the weak topology.

For $f \in U_{\alpha, \beta, a}^{\prime}$, the generalized finite Hankel type transform of $f$ is defined by

$F(n)=<f(x), x J_{\alpha-\beta}\left(\lambda_{n} x\right)>$, for $n=0,1,2, \ldots \ldots$ 


\section{B.B.Waphare}

Our main objective in this paper is to define the finite Hankel type transformation $h_{\alpha, \beta}$ on new spaces of generalized functions by developing a new procedure.

We introduce the finite Hankel type transformation $h_{\alpha, \beta}^{*}$ by

$$
\left(h_{\alpha, \beta}^{*} f\right)(n)=\frac{2}{J_{3 \alpha+\beta}^{2}\left(\lambda_{n}\right)} \int_{0}^{1} J_{\alpha-\beta}\left(\lambda_{n} x\right) f(x) d x, \quad n=0,1,2, \ldots \ldots .
$$

when $(\alpha-\beta) \geq-\frac{1}{2}$.

The transformation $h_{\alpha, \beta}$ and $h_{\alpha, \beta}^{*}$ are closely connected and they satisfy the Parseval equation

$\sum_{n=0}^{\infty}\left(h_{\alpha, \beta} f\right)(n)\left(h_{\alpha, \beta}^{*} \phi\right)(n)=\int_{0}^{1} f(x) \phi(x) d x$

when $(\alpha-\beta) \geq-\frac{1}{2}$ and $f$ and $\phi$ are suitable functions.

We define a space $S_{\alpha, \beta}$ of functions and a space $L_{\alpha, \beta}$ of sequences and we prove that $h_{\alpha, \beta}^{*}$ is an isomorphism from $S_{\alpha, \beta}$ onto $L_{\alpha, \beta}$ provided that $(\alpha-\beta) \geq-\frac{1}{2}$. The generalized finite Hankel type transformation $h_{\alpha, \beta} f$ of $f \in S_{\alpha, \beta}^{\prime}$, the dual of $S_{\alpha, \beta}$, is defined through

$$
<\left(h_{\alpha, \beta}^{\prime} f\right),\left(\left(h_{\alpha, \beta}^{*} \phi\right)(n)\right)_{n=0}^{\infty}>=<f, \phi>\text {, for } \phi \in S_{\alpha, \beta} \text {. }
$$

One can notice that (1.3) appears as a generalization of the Parseval equation (1.2).

We show that the conventional finite Hankel type transformation $h_{\alpha, \beta}$ and generalized finite Hankel type transformation given by (1.1) are special cases of our generalized transformation.

Throughout this paper $(\alpha-\beta)$ denotes a real number greater or equal to $-\frac{1}{2}$.

Now require some properties of Bessel functions.

The behaviours of $J_{\alpha-\beta}$ near the origin and the infinity are the following ones:

$$
\begin{aligned}
J_{\alpha-\beta}(x)=O\left(x^{\alpha-\beta}\right) \text {, as } x \rightarrow 0^{+} & \\
J_{\alpha-\beta}(x) \simeq\left(\frac{2}{\pi x}\right)^{\alpha+\beta} & {\left[\operatorname{Cos}\left(x-\frac{1}{2}(\alpha-\beta) \pi-\frac{\pi}{4} \sum_{m=0}^{\infty} \frac{(-1)^{m}(\alpha-\beta, 2 m)}{(2 x)^{2 m}}\right)\right.} \\
& \left.\quad-\operatorname{Sin}\left(x-\frac{1}{2}(\alpha-\beta) \pi-\frac{1}{4} \pi \sum_{m=0}^{\infty} \frac{(-1)^{m}(\alpha-\beta, 2 m+1)}{(2 x)^{2 m+1}}\right)\right],
\end{aligned}
$$

as $x \rightarrow \infty$,

where $(\alpha-\beta, k)$ is understood as in Watson [17, p.198]

The main differentiation formulae for $J_{\alpha-\beta}$ are

$\frac{d}{d x}\left(x^{\alpha-\beta} J_{\alpha-\beta}(x y)\right)=y x^{\alpha-\beta} J_{-\alpha-3 \beta}(x y)$,

$\frac{d}{d x}\left(x^{-(\alpha-\beta)} J_{\alpha-\beta}(x y)=-y x^{-(\alpha-\beta)} J_{3 \alpha+\beta}(x y)\right)$,

for $x, y>0$. By combining (1.6) and (1.7), it can be easily inferred

$B_{\alpha, \beta} J_{\alpha-\beta}(x)=-J_{\alpha-\beta}(x)$, for $x>0$,

where $B_{\alpha, \beta}=x^{-(3 \alpha+\beta)} D x^{4 \alpha} D x^{-(\alpha-\beta)}$.

\section{The SPACES $S_{\alpha, \beta} A N D L_{\alpha, \beta}$ AND The Finite HANKel TyPe Transformation}

Definition 2.1: We define the $S_{\alpha, \beta}$ as the space of all complex valued functions $\phi(x)$ on $(0,1]$ such that $\phi(x)$ is infinitely differentiable and satisfies for every $k \in \mathbb{N}$ 
(i) $B_{\alpha, \beta}^{* *} \phi(1)=0$,

(ii) $\quad x^{3 \alpha+\beta} B_{\alpha, \beta}^{* k} \phi(x) \rightarrow 0$ and $x^{4 \alpha} D\left(x^{-(3 \alpha+\beta)} B_{\alpha, \beta}^{* k} \phi(x)\right) \rightarrow 0$, as $x \rightarrow 0^{+}$, and

(iii) $\quad x^{-(\alpha+\beta)} B_{\alpha, \beta}^{* k} \phi(x) \in L(0,1)$.

$S_{\alpha, \beta}$ is endowed with the topology generated by the family of seminorms $\left\{\|\phi\|_{k}\right\}_{k=0}^{\infty}$, where

$$
\|\phi\|_{k}=\int_{0}^{1} x^{-(\alpha+\beta)}\left|B_{\alpha, \beta}^{* k} \phi(x)\right| d x, \text { for } \phi \in S_{\alpha, \beta} \text { and } k \in \mathbb{N} .
$$

Notice that $\|\phi\|_{k}$ is a norm for $k=0 . S_{\alpha, \beta}$ is a Hausdorff topological linear space that verifies the first countability axiom. Moreover, the operator $B_{\alpha, \beta}^{*}$ defines a continuous mapping from $S_{\alpha, \beta}$ into itself. $S_{\alpha, \beta}^{\prime}$ is the dual space of $S_{\alpha, \beta}$ and it is equipped with the usual weak topology.

We require the following result which will be useful in the sequel.

Lemma 2.2: If $f(x)$ is a function defined on $(0,1)$ such that $x^{\alpha+\beta} f(x)$ is bounded on $(0,1)$, then $f(x)$ generates a member of $S_{\alpha, \beta}^{\prime}$ through the definition

$$
<f(x), \phi(x)>=\int_{0}^{1} f(x) \phi(x) d x, \phi \in S_{\alpha, \beta} .
$$

Proof: The result easily follows from the following inequality

$$
|<f(x), \phi(x)>| \leq\|\phi\|_{0} \operatorname{Sup}_{0<x<1}\left|x^{\alpha+\beta} f(x)\right|, \phi \in S_{\alpha, \beta} .
$$

Lemma 2.3: Let $(\alpha-\beta) \geq-1 / 2$ and $a \geq 1 / 2$. Then $S_{\alpha, \beta} \subset U_{\alpha, \beta, a}$ and the topology of $S_{\alpha, \beta}$ is stronger than that induced on it by $U_{\alpha, \beta, a}$.

Proof: Let $\phi \in S_{\alpha, \beta}$. In view of the conditions (i) and

(ii) of Definition 2.1, we can write

$$
x^{a-1} B_{\alpha, \beta}^{* k} \phi(x)=x^{a+\alpha-\beta} \int_{1}^{x} t^{-4 \alpha} \int_{0}^{t} u^{\alpha-\beta} B_{\alpha, \beta}^{* k+1} \phi(u) d u d t
$$

for every $x \in(0,1)$ and $k \in \mathbb{N}$.

Therefore

$\left|x^{a-1} B_{\alpha, \beta}^{* k} \phi(x)\right| \leq x^{a+\alpha-\beta} \int_{x}^{1} t^{2 \beta-1} d t \int_{0}^{1} u^{-(\alpha+\beta)}\left|B_{\alpha, \beta}^{* k+1} \phi(u)\right| d \mu$

$\leq x^{a-(\alpha+\beta)} \int_{0}^{1} u^{-(\alpha+\beta)}\left|B_{\alpha, \beta}^{* k+1} \phi(u)\right| d u$

$\leq \int_{0}^{1} u^{-(\alpha+\beta)}\left|B_{\alpha, \beta}^{* k+1} \phi(u)\right| d u$, for every $x \in(0,1)$ and $k \in \mathbb{N}$.

Hence, for every $\phi \in S_{\alpha, \beta}$ and $k \in \mathbb{N}$,

$\operatorname{Sup}_{0<x<1}\left|x^{a-1} B_{\alpha, \beta}^{* k} \phi(x)\right| \leq\|\phi\|_{k+1}$,

and $S_{\alpha, \beta}$ is contained in $U_{\alpha, \beta, a}$ and the inclusion is continuous. Thus proof is completed.

Remark: From Lemma 2.3, we can deduce that if $f \in U_{\alpha, \beta, a}^{\prime}$, then the restriction of $f$ to $S_{\alpha, \beta}$ is a member of $S_{\alpha, \beta}^{\prime}$. 


\section{B.B.Waphare}

Definition 2.4: We define $L_{\alpha, \beta}$ as the space of all complex sequences $\left(a_{n}\right)_{n=0}^{\infty}$ such that $\lim _{n \rightarrow \infty} a_{n} \lambda_{n}^{2 k}=0$, for every $k \in \mathbb{N}$, where $\lambda_{n}, n=0,1,2, \ldots$. represent the positive roots of the equation $J_{\alpha-\beta}(x)=0$ arranged in ascending order of magnitude.

The topology of $L_{\alpha, \beta}$ is that generated by the family of norms $\left\{\gamma_{\alpha, \beta}^{k}\right\}_{k=0}^{\infty}$, where

$$
\gamma_{\alpha, \beta}^{k}\left(\left(a_{n}\right)_{n=0}^{\infty}\right)=\sum_{n=0}^{\infty}\left|a_{n}\right| \lambda_{n}^{2 k}, \text { for }\left(\left(a_{n}\right)_{n=0}^{\infty}\right) \in L_{\alpha, \beta} \text { and } k \in \mathbb{N} .
$$

Notice that $\gamma_{\alpha, \beta}^{k}\left(\left(a_{n}\right)_{n=0}^{\infty}\right)<\infty$ for every $\left(a_{n}\right)_{n=0}^{\infty} \in L_{\alpha, \beta}$.

Thus $L_{\alpha, \beta}$ is Hausdorff topological linear space that satisfies the first countability axiom. $L_{\alpha, \beta}^{\prime}$ denotes the dual space of $L_{\alpha, \beta}$ and it is endowed with the weak topology.

Now we introduce continuous operations in $L_{\alpha, \beta}$ and $L_{\alpha, \beta}^{\prime}$ in the following Lemma.

Lemma 2.5: Let $\left(b_{n}\right)_{n=0}^{\infty}$ be a complex sequence such that $\left|b_{n}\right| \leq M \lambda_{n}^{l}$ for every $n \in \mathbb{N}$ and for some $l \in \mathbb{N}$ and $m>0$.

Then the linear operator

$$
\left(a_{n}\right)_{n=0}^{\infty} \rightarrow\left(a_{n} b_{n}\right)_{n=0}^{\infty}
$$

is a continuous mapping from $L_{\alpha, \beta}$ into itself.

Moreover the operator in $L_{\alpha, \beta}^{\prime}, B \rightarrow\left(b_{n}\right)_{n=0}^{\infty} B$, where

$$
\left\langle\left(b_{n}\right)_{n=0}^{\infty} B,\left(a_{n}\right)_{n=0}^{\infty}\right\rangle=\left\langle B, \quad\left(a_{n} b_{n}\right)_{n=0}^{\infty}\right\rangle, \quad \text { for }\left(a_{n}\right)_{n=0}^{\infty} \in L_{\alpha, \beta},
$$

is a continuous mapping from $L_{\alpha, \beta}^{\prime}$ into itself.

Proof: It is enough to see that

$$
\gamma_{\alpha, \beta}^{k}\left(\left(a_{n} b_{n}\right)_{n=0}^{\infty}\right) \leq M \sum_{n=0}^{\infty}\left|a_{n}\right| \lambda_{n}^{2 k+l} \leq M_{1} \gamma_{\alpha, \beta}^{k+l}\left(\left(a_{n}\right)_{n=0}^{\infty}\right),
$$

for $\left(a_{n}\right)_{n=0}^{\infty} \in L_{\alpha, \beta}$ and $k \in \mathbb{N}$, where $M_{1}$ being a suitable positive constant. This completes the proof.

Lemma 2.6: If $\left(b_{n}\right)_{n=0}^{\infty}$ is a complex sequence satisfying the same conditions as in Lemma 2.5, then $\left(b_{n}\right)_{n=0}^{\infty}$ generates a member of $L_{\alpha, \beta}^{\prime}$ by

$$
\left\langle\left(b_{n}\right)_{n=0}^{\infty},\left(a_{n}\right)_{n=0}^{\infty}\right\rangle=\sum_{n=0}^{\infty} a_{n} b_{n}, \text { for }\left(a_{n}\right)_{n=0}^{\infty} \in L_{\alpha, \beta} .
$$

Now we state our main theorem of this section.

Theorem 2.7: For $(\alpha-\beta) \geq-\frac{1}{2}$, the finite Hankel type transformation $h_{\alpha, \beta}^{*}$ is an isomorphism from $S_{\alpha, \beta}$ onto $L_{\alpha, \beta}$.

Proof: Let $\phi \in S_{\alpha, \beta}$. As it is known, $h_{\alpha, \beta}^{*} \phi=\left(a_{n}\right)_{n=0}^{\infty}$, where

$a_{n}=\frac{2}{J_{3 \alpha+\beta}^{2}\left(\lambda_{n}\right)} \int_{0}^{1} J_{\alpha-\beta}\left(\lambda_{n} x\right) \phi(x) d x$, for every $n \in \mathbb{N}$.

In view of the operational rule (1.6), we can write for every $n \in \mathbb{N}$

$$
\begin{aligned}
\lambda_{n}^{2} a_{n} & =\frac{2 \lambda_{n}^{2}}{J_{3 \alpha+\beta}^{2}\left(\lambda_{n}\right)} \int_{0}^{1} J_{\alpha-\beta}\left(\lambda_{n} x\right) \phi(x) d x \\
& =\frac{2 \lambda_{n}}{J_{3 \alpha+\beta}^{2}\left(\lambda_{n}\right)} \int_{0}^{1} \frac{d}{d x}\left(x^{3 \alpha+\beta} J_{3 \alpha+\beta}\left(\lambda_{n} x\right)\right) x^{-(3 \alpha+\beta)} \phi(x) d x
\end{aligned}
$$


$=\frac{2 \lambda_{n}}{J_{3 \alpha+\beta}^{2}\left(\lambda_{n}\right)}\left[\left(J_{3 \alpha+\beta}\left(\lambda_{n} x\right) \phi(x)\right)_{0}^{1}-\int_{0}^{1} x^{3 \alpha+\beta} J_{3 \alpha+\beta}\left(\lambda_{n} x\right) \frac{d}{d x}\left(x^{-(3 \alpha+\beta)} \phi(x)\right) d x\right]$.

However, by (1.4), $\left(J_{3 \alpha+\beta}\left(\lambda_{n} x\right) \phi(x)\right]_{0}^{1}=0$, since $\phi(1)=0$ and

Hence

$$
\lim _{x \rightarrow 0^{+}} x^{3 \alpha+\beta} \phi(x)=0 \text {. }
$$

$\lambda_{n}^{2} a_{n}=\frac{2 \lambda_{n}}{J_{3 \alpha+\beta}^{2}\left(\lambda_{n}\right)} \int_{0}^{1} x^{3 \alpha+\beta} J_{3 \alpha+\beta}\left(\lambda_{n} x\right) \frac{d}{d x}\left(x^{-(3 \alpha+\beta)} \phi(x)\right) d x$.

Now, by invoking (1.7), we have

$$
\begin{aligned}
& \lambda_{n} \int_{0}^{1} x^{3 \alpha+\beta} J_{3 \alpha+\beta}\left(\lambda_{n} x\right) \frac{d}{d x}\left(x^{-(3 \alpha+\beta)} \phi(x)\right) d x \\
& =-\int_{0}^{1} \frac{d}{d x}\left(x^{-(\alpha-\beta)} J_{\alpha-\beta}\left(\lambda_{n} x\right)\right) x^{4 x} \frac{d}{d x}\left(x^{-(3 \alpha+\beta)} \phi(x)\right) d x \\
& =\left[-J_{\alpha-\beta}\left(\lambda_{n} x\right) x^{3 \alpha+\beta} \frac{d}{d x}\left(x^{-(3 \alpha+\beta)} \phi(x)\right)\right]_{0}^{1}+\int_{0}^{1} B_{\alpha, \beta}^{*} \phi(x) J_{\alpha-\beta}\left(\lambda_{n} x\right) d x .
\end{aligned}
$$

The limit terms are equal to zero by (1.4) because $J_{\alpha-\beta}\left(\lambda_{n}\right)=0, \phi \in C^{\infty}((0,1])$,

$$
\lim _{x \rightarrow 0^{+}} x^{4 \alpha} \frac{d}{d x}\left(x^{-(3 \alpha+\beta)} \phi(x)\right)=0 .
$$

Therefore

$\lambda_{n} \int_{0}^{1} x^{3 \alpha+\beta} J_{3 \alpha+\beta}\left(\lambda_{n} x\right) \frac{d}{d x}\left(x^{-(3 \alpha+\beta)} \phi(x)\right) d x=\int_{0}^{1} B_{\alpha, \beta}^{*} \phi(x) J_{\alpha, \beta}\left(\lambda_{n} x\right) d x$

Using relations (2.1) and (2.2), we obtain

$$
a_{n} \lambda_{n}^{2}=-\frac{2}{J_{3 \alpha+\beta}^{2}\left(\lambda_{n}\right)} \int_{0}^{1} B_{\alpha, \beta}^{*} \phi(x) J_{\alpha-\beta}\left(\lambda_{n} x\right) d x \text {, for every } n \in \mathbb{N} .
$$

By induction, we have

$\lambda_{n}^{2 k} a_{n}=(-1)^{k} \frac{2}{J_{3 \alpha+\beta}^{2}\left(\lambda_{n}\right)} \int_{0}^{1} B_{\alpha, \beta}^{* k} \phi(x) J_{\alpha-\beta}\left(\lambda_{n} x\right) d x$, for every $n, k \in \mathbb{N}$.

From (2.3), according to Riemann-Lebesgue Lemma ([17, p. 457]), we have

$$
J_{3 \alpha+\beta}^{2}\left(\lambda_{n}\right) \lambda_{n}^{2 k} a_{n} \rightarrow 0 \text {, as } n \rightarrow \infty .
$$

Moreover by (1.5), there exists a positive constant $M$ such that

$$
\lambda_{n}^{2 k}\left|a_{n}\right| \leq M J_{3 \alpha+\beta}^{2}\left(\lambda_{n}\right) \lambda_{n}^{2 k+1}\left|a_{n}\right|,
$$

and then $\lambda_{n}^{2 k} a_{n} \rightarrow 0$, as $n \rightarrow \infty$, for every $k \in \mathbb{N}$.

Now, for certain $M_{i}, i=0,1,2$,

$$
\begin{aligned}
& \sum_{n=0}^{\infty} \lambda_{n}^{2 k}\left|a_{n}\right|=\sum_{n=0}^{\infty} \frac{2}{J_{3 \alpha+\beta}^{2}\left(\lambda_{n}\right) \lambda_{n}^{4}}\left|\int_{0}^{1} B_{\alpha, \beta}^{* k+2} \phi(x) J_{\alpha-\beta}\left(\lambda_{n} x\right) d x\right| \\
& \leq M_{1} \sum_{n=0}^{\infty} \lambda_{n}^{-5(\alpha+\beta)} \int_{0}^{1}\left|\left(\lambda_{n} x\right)^{\alpha+\beta} J_{\alpha-\beta}\left(\lambda_{n} x\right)\right| x^{-(\alpha+\beta)}\left|B_{\alpha, \beta}^{* k+2} \phi(x)\right| d x \\
& \leq M_{2} \sum_{n=0}^{\infty} \lambda_{n}^{-2} \int_{0}^{1} x^{-(\alpha+\beta)}\left|B_{\alpha, \beta}^{* k+2} \phi(x)\right| d x .
\end{aligned}
$$




\section{B.B.Waphare}

Since

$$
\sum_{n=0}^{\infty} \lambda_{n}^{-2}<\infty
$$

we get

$\gamma_{\alpha, \beta}^{k}\left(\left(a_{n}\right)_{n=0}^{\infty}\right) \leq M_{3}\|\phi\|_{k+2}$, for every $k \in \mathbb{N}$ and $\phi \in S_{\alpha, \beta}$ and for some $M_{3}>0$.

This inequality proves that the linear mapping $h_{\alpha, \beta}^{*}$ is continuous from $S_{\alpha, \beta}$ into $L_{\alpha, \beta}$.

Now let $\left(a_{n}\right)_{n=0}^{\infty} \in L_{\alpha, \beta}$ and define

$$
\tau_{\alpha, \beta}\left(\left(a_{n}\right)_{n=0}^{\infty}\right)(x)=\phi(x)=\sum_{n=0}^{\infty} a_{n} x J_{\alpha-\beta}\left(\lambda_{n} x\right), \text { for } x \in(0,1] .
$$

By (1.4) and (1.5), we have

$$
\sum_{n=0}^{\infty}\left|a_{n} x J_{\alpha-\beta}\left(\lambda_{n} x\right)\right| \leq M x^{\alpha+\beta} \sum_{n=0}^{\infty}\left|a_{n}\right|, x>0
$$

for a suitable $M>0$. Thus $\phi(x) \in C(0, \infty)$. In a similar way we can prove that $\phi \in C^{\infty}(0, \infty)$. Moreover by invoking (1.8) we obtain

and $k \in \mathbb{N}$.

$$
B_{\alpha, \beta}^{* k} \phi(x)=\sum_{n=0}^{\infty}(-1)^{k} a_{n} \lambda_{n}^{2 k} x J_{\alpha-\beta}\left(\lambda_{n} x\right), \text { for } x>0
$$

Then $B_{\alpha, \beta}^{* k} \phi(1)=0$, for each $k \in \mathbb{N}$.

We can also infer

$$
\left|x^{3 \alpha+\beta} B_{\alpha, \beta}^{* k} \phi(x)\right| \leq M_{1} x^{2(2 \alpha+\beta)} \sum_{n=0}^{\infty}\left|a_{n}\right| \lambda_{n}^{2 k}, \text { for } x>0 \text { and } k \in \mathbb{N},
$$

and from (1.4), (1.5) and (1.6),

$$
\left|x^{4 \alpha} \frac{d}{d x}\left(x^{-(3 \alpha+\beta)} B_{\alpha, \beta}^{* k} \phi(x)\right)\right| \leq M_{2} x^{2(3 \alpha+\beta)} \sum_{n=0}^{\infty}\left|a_{n}\right| \lambda_{n}^{2 k+5 \alpha+3 \beta},
$$

for $x>0$ and $k \in \mathbb{N}$.

Here $M_{1}$ and $M_{2}$ are suitable positive constants. Hence

$$
\lim _{x \rightarrow 0^{+}} x^{3 \alpha+\beta} B_{\alpha, \beta}^{* k} \phi(x)=\lim _{x \rightarrow 0^{+}} x^{4 \alpha} \frac{d}{d x}\left(x^{-(3 \alpha+\beta)} B_{\alpha, \beta}^{* k} \phi(x)\right)=0,
$$

for every $k \in \mathbb{N}$.

On the other hand, as the series defining $B_{\alpha, \beta}^{* k} \phi(x)$ is uniformly convergent in $x \in(0,1)$, there exists a positive constant $M_{3}$ such that

$$
\int_{0}^{1} x^{-(\alpha+\beta)}\left|B_{\alpha, \beta}^{* k} \phi(x)\right| d x \leq M_{3} \sum_{n=0}^{\infty}\left|a_{n}\right| \lambda_{n}^{2 k},
$$

for every $k \in \mathbb{N}$.

Therefore $\tau_{\alpha, \beta}$ is a continuous mapping from $L_{\alpha, \beta}$ into $S_{\alpha, \beta}$. Finally from Watson [17,p.59], we can infer that

$$
\begin{aligned}
& \left(\tau_{\alpha, \beta} \cdot h_{\alpha, \beta}^{*}\right) \phi=\phi, \text { for } \phi \in S_{\alpha, \beta} \text {, and } \\
& \left(h_{\alpha, \beta}^{*} \cdot \tau_{\alpha, \beta}\right)\left(a_{m}\right)_{n=0}^{\infty}=\left(a_{m}\right)_{n=0}^{\infty}, \text { for }\left(a_{n}\right)_{n=0}^{\infty} \in L_{\alpha, \beta} .
\end{aligned}
$$

This completes the proof. 


\section{The Generalized Finite HANKEL TYPE TranSFORMATION}

We define the generalized finite Hankel type transformation $h_{\alpha, \beta}^{\prime}$ on $S_{\alpha, \beta}^{\prime}$ as follows.

$\left\langle\left(h_{\alpha, \beta}^{\prime} f\right),\left(h_{\alpha, \beta}^{*} \phi\right)(n)_{n=0}^{\infty}\right\rangle=\langle f(x), \phi(x)\rangle$

for every $\phi \in S_{\alpha, \beta}$. Notice that (3.1) appears as a generalization of the Parseval equation (1.2).

From Zemanian [19, Theorem 1.10-2] and Theorem 2.7, we immediately obtain

Theorem 3.1: For $(\alpha-\beta) \geq-1 / 2$, the generalized finite Hankel type transformation $h_{\alpha, \beta}^{\prime}$ is an isomorphism from $S_{\alpha, \beta}^{\prime}$ onto $L_{\alpha, \beta}^{\prime}$.

In the following theorem, we establish that the conventional finite Hankel type transformation $h_{\alpha, \beta}$ is a special case of the generalized finite Hankel type transformation defined in (3.1).

Theorem 3.2: Let $f(x)$ be a function defined on $(0,1)$ such that $x^{\alpha+\beta} f(x)$ is bounded on $(0,1)$. Then $\left(\left(h_{\alpha, \beta} f\right)(n)\right)_{n=0}^{\infty}$ agrees with $\left(h_{\alpha, \beta}^{\prime} f\right)$ as members of $L_{\alpha, \beta}^{\prime}$.

Proof: The conventional finite Hankel type transformation of $f$ is defined by

$$
\left(h_{\alpha, \beta} f\right)(n)=\int_{0}^{\prime} x J_{\alpha-\beta}\left(\lambda_{n} x\right) f(x) d x \text {, for } n \in \mathbb{N} .
$$

Then as $x^{\alpha+\beta} f(x)$ is bounded on $(0,1)$ and by (1.4) and (1.5) we can write

$$
\begin{aligned}
\left|\left(h_{\alpha, \beta} f\right)(n)\right| & \leq M \lambda_{n}^{-(\alpha+\beta)} \int_{0}^{1}\left|\left(\lambda_{n} x\right)^{\alpha+\beta} J_{\alpha-\beta}\left(\lambda_{n} x\right)\right| d x \\
& \leq M_{1} \lambda_{n}^{-(\alpha+\beta)}, \quad \text { for } n \in \mathbb{N},
\end{aligned}
$$

where $M$ and $M_{1}$ are certain positive constants.

Therefore in view of Lemma 2.6, $\left(\left(h_{\alpha, \beta} f\right)(n)\right)_{n=0}^{\infty}$ generates a member of $L_{\alpha, \beta}^{\prime}$ by

$$
\begin{aligned}
\left\langle\left(\left(h_{\alpha, \beta} f\right)(n)\right)_{n=0}^{\infty},\left(a_{n}\right)_{n=0}^{\infty}\right\rangle=\sum_{n=0}^{\infty} & \left(h_{\alpha, \beta} f\right)(n) a_{n} \\
& =\sum_{n=0}^{\infty} a_{n} \int_{0}^{1} x J_{\alpha-\beta}\left(\lambda_{n} x\right) f(x) d x \\
& =\int_{0}^{1} f(x) \sum_{n=0}^{\infty} a_{n} x J_{\alpha-\beta}\left(\lambda_{n} x\right) d x
\end{aligned}
$$

for every $\left(a_{n}\right)_{n=0}^{\infty} \in L_{\alpha, \beta}$

The last equality is justified since the series

$$
\sum_{n=0}^{\infty} a_{n} x^{\alpha+\beta} J_{\alpha-\beta}\left(\lambda_{n} x\right)
$$

is uniformly convergent on $(0,1)$ and $x^{\alpha+\beta} f(x)$ is bounded on $(0,1)$.

We can also write

$\left\langle\left(\left(h_{\alpha, \beta} f\right)(n)\right)_{n=0}^{\infty},\left(\left(h_{\alpha, \beta} \phi\right)(n)\right)_{n=0}^{\infty}\right\rangle$ 
$=\int_{0}^{1} f(x) \sum_{n=0}^{\infty}\left(h_{\alpha, \beta}^{*} \phi\right)(n) x J_{\alpha-\beta}\left(\lambda_{n} x\right) d x=\int_{0}^{1} f(x) \phi(x) d x$

for every $\phi \in S_{\alpha, \beta}$.

Hence according Lemma 2.2, we conclude

$\left\langle\left(\left(h_{\alpha, \beta} f\right)(n)\right)_{n=0}^{\infty},\left(\left(h_{\alpha, \beta}^{*} \phi\right)(n)\right)_{n=0}^{\infty}\right\rangle=<f(x), \phi(x)$, for $\phi \in S_{\alpha, \beta}$ and $\left(\left(h_{\alpha, \beta} f\right)(n)\right)_{n=0}^{\infty}=$ $\left(h_{\alpha, \beta}^{\prime} f\right)$ as members of $L_{\alpha, \beta}^{\prime}$. Thus proof is completed.

We now prove that the generalized finite Hankel type transform of $f$ given by (1.1) is equal (in the sense of equality in $L_{\alpha, \beta}^{\prime}$ ) to the generalized finite Hankel type transform of $f$ as given by (3.1).

Theorem 3.3: Let $(\alpha-\beta) \geq-1 / 2, a \geq 1 / 2$ and $f \in U_{\alpha, \beta, a}^{\prime}$.

Then

$\left\langle(F(n))_{n=0}^{\infty},\left(a_{n}\right)_{n=0}^{\infty}\right\rangle=\left\langle\left(h_{\alpha, \beta}^{\prime} f\right),\left(a_{n}\right)_{n=0}^{\infty}\right\rangle$, for every $\left(a_{n}\right)_{n=0}^{\infty} \in L_{\alpha, \beta}^{\prime}$,

where,

$$
F(n)=\left\langle f(x), \quad x J_{\alpha-\beta}\left(\lambda_{n} x\right)\right\rangle, \quad \text { for every } n \in \mathbb{N} .
$$

Proof: By Zemanian [19, Theorem 1.8-1], since $\in U_{\alpha, \beta, a}^{\prime}$, there exist $r \in \mathbb{N}$ and $M>0$ such that

$$
\left|\left\langle f(x), \quad x J_{\alpha-\beta}\left(\lambda_{n} x\right)\right\rangle\right| \leq M \max _{0 \leq k \leq r} \operatorname{Sup}_{0<x<1}\left|x^{a-1} B_{\alpha, \beta}^{* k}\left(x J_{\alpha-\beta}\left(\lambda_{n} x\right)\right)\right|,
$$

for every $n \in \mathbb{N}$.

Hence, by (1.4), (1.5) and (1.8), we can infer that

$|F(n)| \leq M \max _{0 \leq k \leq r} \operatorname{Sup}_{0<x<1}\left|x^{a-1} \lambda_{n}^{2 k} x J_{\alpha-\beta}\left(\lambda_{n} x\right)\right| \leq M_{1} \lambda_{n}^{2 r}$

for a certain $M_{1}>0$. By invoking Lemma 2.6, (3.2) proves that $(F(n))_{n=0}^{\infty}$ generates a member of $L_{\alpha, \beta}^{\prime}$ through

$$
\left\langle(F(n))_{n=0}^{\infty}, \quad\left(a_{n}\right)_{n=0}^{\infty}\right\rangle=\sum_{n=0}^{\infty} F(n) a_{n}, \text { for }\left(a_{n}\right)_{n=0}^{\infty} \in L_{\alpha, \beta} .
$$

To prove our assertion, we must establish that

$\sum_{n=0}^{\infty} F(n) a_{n}=\left\langle f(x), \sum_{n=0}^{\infty} a_{n} x J_{\alpha-\beta}\left(\lambda_{n} x\right)\right\rangle$, for $\left(a_{n}\right)_{n=0}^{\infty} \in L_{\alpha, \beta}$.

Let $\left(a_{n}\right)_{n=0}^{\infty} \in L_{\alpha, \beta}$. As it is easy to see,

$\sum_{n=0}^{\infty} F(n) a_{n}=\left\langle f(x), \sum_{n=0}^{m} a_{n} x J_{\alpha-\beta}\left(\lambda_{n} x\right)\right\rangle+\sum_{n=m+1}^{\infty} a_{n}\left\langle f(x), x J_{\alpha-\beta}\left(\lambda_{n} x\right)\right\rangle$.

for every $m \in \mathbb{N}$.

By using (3.2), we can obtain

$$
\left|\sum_{n=m+1}^{\infty} a_{n}\left\langle f(x), \quad x J_{\alpha-\beta}\left(\lambda_{n} x\right)\right\rangle\right| \leq M_{1} \sum_{n=m+1}^{\infty}\left|a_{n}\right| \lambda_{n}^{2 r},
$$

for every $m \in \mathbb{N}$ with $M_{1}>0$. Then

$\lim _{m \rightarrow \infty} \sum_{n=m+1}^{\infty} a_{n}\left\langle f(x), x J_{\alpha-\beta}\left(\lambda_{n} x\right)\right\rangle=0$.

Moreover, for every $k \in \mathbb{N}$ and $x \in(0,1)$, we obtain

$\left|x^{a-1} B_{\alpha, \beta}^{* k}\left[\sum_{n=m+1}^{\infty} a_{n} x J_{\alpha-\beta}\left(\lambda_{n} x\right)\right]\right|$ 
$\leq x^{a-1} \sum_{n=m+1}^{\infty}\left|a_{n} x J_{\alpha-\beta}\left(\lambda_{n} x\right)\right| \lambda_{n}^{2 k} \leq M_{2} x^{a-(\alpha+\beta)} \sum_{n=m+1}^{\infty}\left|a_{n}\right| \lambda_{n}^{2 k}$

for a suitable $M_{2}>0$.

Hence

for every $k \in \mathbb{N}$, and

$$
\operatorname{Sup}_{0<x<1}\left|x^{a-1} B_{\alpha, \beta}^{* k}\left[\sum_{n=m+1}^{\infty} a_{n} x J_{\alpha-\beta}\left(\lambda_{n} x\right)\right]\right| \leq M_{2} \sum_{n=m+1}^{\infty}\left|a_{n}\right| \lambda_{n}^{2 k},
$$

$$
\sum_{n=m+1}^{\infty} a_{n} x J_{\alpha-\beta}\left(\lambda_{n} x\right) \rightarrow 0, \text { as } m \rightarrow \infty, \quad \text { in } S_{\alpha, \beta},
$$

because $\left(a_{n}\right)_{n=0}^{\infty} \in L_{\alpha, \beta}$.

Therefore, since $f \in S_{\alpha, \beta}^{\prime}$,

$\lim _{m \rightarrow \infty}\left\langle f(x), \quad \sum_{n=m+1}^{\infty} a_{n} x J_{\alpha-\beta}\left(\lambda_{n} x\right)\right\rangle=0$.

Now from (3.3), we can conclude

$$
\begin{aligned}
& \left\langle(F(n))_{n=0}^{\infty},\left(\left(h_{\alpha, \beta}^{*} \phi\right)(n)\right)_{n=0}^{\infty}\right\rangle=\left\langle f(x), \sum_{n=0}^{\infty}\left(h_{\alpha, \beta}^{*} \phi\right)(m) x J_{\alpha-\beta}\left(\lambda_{n} x\right)\right\rangle \\
& =\langle f(x), \phi(x)\rangle=\left\langle\left(h_{\alpha, \beta}^{\prime} f\right),\left(\left(h_{\alpha, \beta}^{*} \phi\right)(n)\right)_{n=0}^{\infty}, \quad \text { for } \phi \in S_{\alpha, \beta},\right\rangle
\end{aligned}
$$

and the proof is complete.

\section{Application}

Firstly we prove an operational-transform formula for the generalized finite Hankel type transformation that will be useful in applications.

Lemma 4.1: Let $P$ be a polynomial and $f$ be in $S_{\alpha, \beta}^{\prime}$. Then

$$
\left(h_{\alpha, \beta}^{\prime} P\left(B_{\alpha, \beta}\right) f\right)=P\left(-\lambda_{n}^{2}\right)\left(h_{\alpha, \beta}^{\prime} f\right) .
$$

Proof: If $f \in S_{\alpha, \beta}^{\prime}$, we have

$$
\begin{aligned}
& \left\langle\left(h_{\alpha, \beta}^{\prime} P\left(B_{\alpha, \beta}\right) f\right), \quad\left(\left(h_{\alpha, \beta}^{*} \phi\right)(n)\right)_{n=0}^{\infty}\right\rangle \\
& =\left\langle P\left(B_{\alpha, \beta}\right) f, \phi\right\rangle=\left\langle f, P\left(B_{\alpha, \beta}^{*}\right) \phi\right\rangle \\
& =\left\langle\left(h_{\alpha, \beta}^{\prime} f\right), \quad\left(\left(h_{\alpha, \beta}^{*} P\left(B_{\alpha, \beta}^{*}\right) \phi\right)(n)\right)_{n=0}^{\infty}\right\rangle \\
& =\left\langle\left(h_{\alpha, \beta}^{\prime} f\right), \quad\left(P\left(-\lambda_{n}^{2}\right)\left(h_{\alpha, \beta}^{*} \phi\right)(n)\right)_{n=0}^{\infty}\right\rangle, \\
& =\left\langle P\left(-\lambda_{n}^{2}\right)\left(h_{\alpha, \beta}^{\prime} f\right), \quad\left(\left(h_{\alpha, \beta}^{*} \phi\right)(n)\right)_{n=0}^{\infty}\right\rangle, \text { for every } \phi \in S_{\alpha, \beta} .
\end{aligned}
$$

We consider the functional equation

$$
P\left(B_{\alpha, \beta}\right) f=g,
$$

where $g$ is a given member of $S_{\alpha, \beta}^{\prime}, P$ is a polynomial such that $P\left(-\lambda_{n}^{2}\right) \neq 0$ for every $n \in \mathbb{N}$, and $f$ is unknown generalized function but required to be in $S_{\alpha, \beta}^{\prime}$.

By applying the generalized finite Hankel type transform to (4.1) and according to Lemma 4.1 , we can prove that (4.1) is equivalent to

$$
P\left(-\lambda_{n}^{2}\right)\left(h_{\alpha, \beta}^{\prime} f\right)=\left(h_{\alpha, \beta}^{*} g\right) .
$$


Hence it is not difficult to see that the functional $f$ defined by

$$
\langle f, \phi\rangle=\left\langle g, \sum_{n=0}^{\infty} \frac{1}{P\left(-\lambda_{n}^{2}\right)}\left(h_{\alpha, \beta}^{*} \phi\right)(n) x J_{\alpha-\beta}\left(\lambda_{n} x\right)\right\rangle, \quad \text { for } \phi \in S_{\alpha, \beta},
$$

is in $S_{\alpha, \beta}^{\prime}$ and it is the solution for (4.1). This completes the proof.

\section{Conclusion}

This paper provides the study of the finite Hankel type transformation on spaces of generalized functions. The integral transformations $h_{\alpha, \beta}$ and $h_{\alpha, \beta}^{*}$ satisfy Parseval type equation defined above in this paper. We have shown that $h_{\alpha, \beta}^{*}$ and $h_{\alpha, \beta}^{\prime}$ are isomorphisms from $S_{\alpha, \beta}$ onto $L_{\alpha, \beta}$ and $S_{\alpha, \beta}^{\prime}$ onto $L_{\alpha, \beta}^{\prime}$ respectively. Applications of new generalized finite Hankel type transformation established in this paper may be useful in engineering.

\section{ACKNOWLEDGEMENT}

Author is thankful to referee for his valuable suggestions in improving this paper.

\section{REFERENCES}

[1] Betancor J.J, The Hankel-Schwartz transform for functions of compact support, Rend. Mat. Appl. 7(3-4) (1987), 399-409.

[2] Betancor J.J., A mixed Parseval equation and a generalized Hankel transformation of distributions, Can. J. Math. XLI (2) (1989), 274-284.

[3] Churchill R.V., Fourier Series and Boundary Value Problems, Mc Graw-Hill, New York, 1953.

[4] Cinelli, A extension of the finite Hankel transform and applications, Inst. J. Engng. 3 (1965), 539-559.

[5] Dube L.S., On finite Hankel-transformation of generalized functions, Pacific, J. Math. 62 (1976), 365-378.

[6] Gelfand I.M., Shilov G.E., Generalized functions, Vol. III. Academic Press, New York, 1967.

[7] Liu S.H., Method of generalized finite Hankel transform, Z. Angew. Math. Mech. 51 (1971), 311-313.

[8] Mendez J.M., A mixed Parseval equation and the generalized Hankel transformation, Proc. Amer. Math. Soc. 102 (1988), 619-624.

[9] Mendez J.M., The finite Hankel-Schwartz transform, J. Korean Math. Soc 26(1) (1989), 647655.

[10] Mendez J.M. and Negrin J.R., Fourier Bessel Series expansion of generalized functions and finite Hankel transforms of distributions, Rev. Roum. de. Math. Pures et. Appl. XXXIV (7) (1989), 647-655.

[11] Pandey J.N., Pathak R.S., Eigen function expansion of generalized functions, Nagoya Math. J.72 (1978), 1-25.

[12] Pathak R.S., Orthogonal series representations for generalized functions, J. Math. Anal. Appl. 130 (1988), 316-333.

[13] Pathak R.S, Singh O.P, Finite Hankel transforms of distributions, Pacific J. Math. 99 (1982), 439-458.

[14] Sneddon I.N., On finite Hankel transforms, Phil. Mag. (7) 17 (1946), 16-25.

[15] Sneddon I.N., The Use of Integral Transforms, Tata Mc Graw. Hill, New Delhi, 1979.

[16] Titchmarsh E.C., A class of expansions in series of Bessel functions, Proc. London Math. Soc. (2) 22 (1924), Xiii-Xvi.

[17] Watson G.N., Theory of Bessel Functions, 2nd.ed., Cambridge University Press, Cambridge, 1958.

[18] Zemanian A. H., Orthonormal series expansions of certain distributions and distributional transform calculus, J. Math. Anal. Appl. 14(1966), 263-275.

[19] Zemanian A. H., Generalized Integral Transformations, Interscience Publishers, New York, 1968. 


\section{AUTHOR'S BIOGRAPHY}

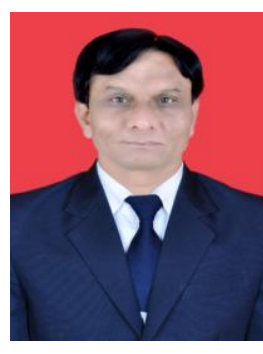

Prof Dr.Balasaheb Bhagaji Waphare, is a science graduate from Fergusson college, Pune in 1989.He has completed his Master and Doctorate degrees from a well known and reputed Savitribai Phule Pune University (Formerly known as University of Pune).Presently he is working as a principal at MAEER Pune's MIT Arts, Commerce \& Science College (MITACSC),Alandi(D).He has wide experience of more than 22 years in teaching and research. He is a research guide in Mathematics recognized by Savitrivbai Phule Pune University for M.Phil and Ph.D programmes.

Dr.Balasaheb Waphare is a very devoted, dedicated and passionate teacher served with distinction. He has 10 awards to his name and attended 25 National and International conferences .He is also a life member of 08 reputed professional bodies and reviewer of few reputed journals.Dr.Balasaheb B.Waphare has more than 90 research publications to his credits in various reputed National and International journals. 